\title{
A TCP TEST SYSTEM SPECIFYING ONLY EXCEPTIONAL PACKET SEQUENCES
}

\author{
Tomohiko Ogishi, Toru Hasegawa, Toshihiko Kato and Kenji Suzuki \\ KDDI R\&D Laboratories, Inc., Japan
}

\begin{abstract}
Although TCP is widely used in Internet, new specifications are still proposed and implemented. In the circumstance above, it is highly possible that some errors are detected on the communication between new and old implementations. Several test tools were developed so far. However, they do not have enough function to customize test sequence or need significant effort to specify the sequence. We developed a TCP test system which specifies only exceptional packet sequence in the test scenario. The system performs exceptional packet sequence only when the condition specified in the test scenario is satisfied. Otherwise, it performs ordinary TCP behavior. The system is implemented by modifying TCP module of NetBSD with SACK code developed by Pittsburgh Supercomputing Center. We also evaluated SACK implementation as an example of recent specification using the test system.
\end{abstract}

Keywords: TCP, exceptional packet sequence, test scenario, congestion control, selective acknowledgment

\section{INTRODUCTION}

TCP [1] is widely used as a protocol to provide reliable transfer from the dawn of Internet. The TCP protocol functions and implementations have been modified and extended in the long history of TCP. Currently, the TCP implementations in widely spread PCs and workstations are fairly stable and the users seldom feel inconvenience at TCP communications. However, there are several implementation errors as reported in [2]. Due to those errors, TCP communications in specific situations, such as a long haul TCP 
communication, suffer from problems like serious throughput degradation. Those well-known errors have been fixed in the higher version of implementations, but, still now, new functions, such as a new congestion control mechanism in NewReno [3] and the selective acknowledgment (SACK) [4], are proposed and integrated into available TCP implementations. It is highly possible that not detected errors related to conventional functions [5] and errors related to new functions $[3,4]$ bring new problems in TCP communications.

In order to detect such errors, some tools for testing TCP implementation are required. So far, various tools have been developed [6], which are classified into two groups, monitors and testers. The monitors collect packet data exchanged between end systems and analyzes TCP protocol behaviors. TCPAnaly [7] and the Intelligent TCP Analyzer [8] are categorized in this group. However, the monitors cannot control packet sequences in the testing, and it is not easy to detect errors in TCP implementations by monitors.

On the other hand, the testers can generate packets suitable for a specific testing purpose. But, the testers developed so far [9-11] have some issues to perform the TCP implementation testing. Among them, TBIT [9] and Nmap [10] use some predefined test sequences. The purpose of TBIT is to check the compliance and used parameter values of TCP implementations, and that of Nmap is to estimate the version of operating systems from the TCP behaviors. That is, those systems do not allow test operators to use test sequences specific to their test purposes. On the other hand, [11] proposes a TCP test system which can generate test sequences based on test scenario specification written in TTCN [12]. This system allows test operators to use suited test sequences. However, the task to generate a test sequence will be a hard job for test operators because it is required to specify all input and output events used in the sequence.

Considering these issues, it is important to reduce the burden for test operators to specify a test sequence. When a test operator tries to test a normal behavior of TCP module in a system under test, it is possible to use an ordinary TCP module as a tester. A specially ordered mechanism is used only when a test operator tries to test an exceptional behavior of TCP. Therefore, we propose a TCP test system which uses an ordinary TCP module for testing normal TCP behaviors and which allows test operators to specify a test sequence only for testing exceptional TCP sequences. By using our TCP test system, a test operator can perform exceptional test sequence, such as sending SYN segment in ESTABLISHED state, sending ACK segment with smaller acknowledgment number, and sending SACK options with the first and the second SACK blocks misordered. A test operator does not need to specify normal sequences but describe exceptional part in a test scenario. The TCP module in the test system behaves normally if the 
condition on a test scenario is not satisfied. If the condition is satisfied, it runs the actions specified in the test scenario. It also saves a communication $\log$ which is examined after the test run is over.

In this paper, we describe the requirements, design and implementation of the TCP test system. We also describe the evaluation result of SACK implementation by using the system. In Section 2, we describe the requirement and the design principles of our test system. Sections 3 and 4 show the detailed design and the implementation, respectively. Section 5 gives an evaluation of SACK implementation by using the test system. Finally, Section 6 makes a conclusion.

\section{REQUIREMENTS AND DESIGN PRINCIPLES}

As described above, the following is required for the TCP test system.

- The test system should provide a convenient method to specify a test scenario in order to reduce the burden for test operator.

- The essential functions of TCP such as connection establishment have been tested sufficiently so far, and are used without any problem. However, in some recent specifications, there are some additional functions which rely on implementers' decision. For example, SACK specifies the format of SACK blocks, but, it does not specify the retransmission manner when a packet with SACK option is received. Therefore, it is such an additional function that should be tested by the TCP test system.

- In the test of additional functions, it is expected that a test operator will examine the test result in detail, estimate the reasons for the result by emulating the TCP behaviors of the system under test, and give the verdict for the test. In order to reduce the burden for the test result analysis, the TCP test system should generate enough information on the communication in the test logs.

Based on these requirements, we have adopted the following design principles in order to develop the TCP test system.

1. The test system is composed by test execution part and test analysis part. The test execution part communicates with system under test using some TCP application program for testing. It performs exceptional behaviors specified in a test scenario while communicating by normal behavior. It collects the communication log for later analysis. The test analysis part analyzes the communication $\log$ in an offline manner.

2. Only the exceptional sequences are described in a test scenario. This means that the TCP test system communicates with system under test 
according to the normal TCP procedure, while it is not directed to follow exceptional sequence. Once it is directed, it sends segments with the parameter values and with the order which are specified in the test scenario.

3. In order to develop the test execution part, we use TCP application program and in-kernel TCP module. We use public software such as ftp or ttcp as a TCP application program. We modify the in-kernel TCP module in order to realize the test scenario execution and the communication log collection. We believe that this implementation scheme requires less amount of work compared with the implementation of the test execution part from the scratch, although our scheme requires the kernel modification.

4. A test scenario is composed by a set of rows, each of which specifies a condition and an action. A condition specifies whether to trigger the corresponding action. We use three types of conditions; the parameter values of received segments, the parameter values of segments going to be sent and the expiration of user-defined timers. The action specifies whether to send segments if the condition is satisfied. We use two type of actions; a sending of a segments with the specific parameter values, and the discard of a segment which is sent by the original TCP module.

\section{DETAILED DESIGN}

\subsection{Structure}

Figure 1 shows the functional structure of the TCP test system. The test system is composed by test execution part and test analysis part as described in the previous section. The test execution part includes scenario loader, TCP application program, in-kernel TCP module and network interface. In the in-kernel TCP module, scenario interpreter and log collector are implemented. Scenario loader provides the operator to configure the test environment. It selects the test scenario performed at scenario interpreter and whether the communication log is collected or not. TCP application program actually sends or receives user data on TCP by communicating with server or client program running on system under test. Scenario interpreter reads the test scenario and decides whether the action in the test scenario is executed or not. When the action is executed, it directly sends the packets described in the action to network interface through log collector. The interpreter maintains the scenario counter indicating the line of test scenario. Log collector is placed on the network interface and monitors the segments 
received from or sent to network interface, and collects a communication log related to the test. It also refers to the scenario counter when a segment is added to the communication log. Log analyzer in the test analysis part provides the operator to analyze the communication log.

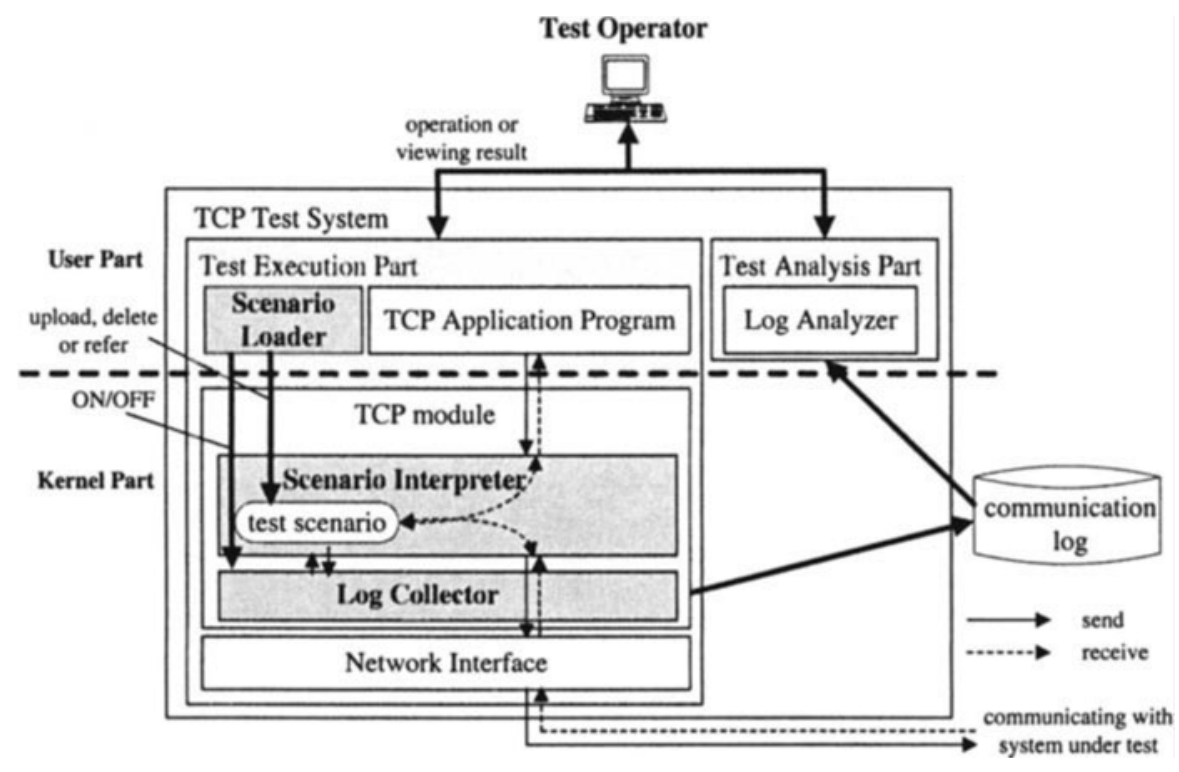

Figure 1. Structure of TCP Test System.

\subsection{Test Scenario}

Figure 2 shows an example of test scenario. The test scenario consists of header and content parts. In the header part, IP addresses and port numbers for source and destination, which indicate TCP connections under test. For example, if 20 is specified as source port number, the TCP behavior of FTP data connections becomes the target. In this case, TCP connections with other source port number such as FTP control connection or WWW client behaves without any influence of test scenario. In other words, the system can protect other application programs from being affected by the test scenario. In the header part, TCP options used at connection establishment and the parameter value of each option are also specified by using syn-opt key. When the key is specified, the specified options are forced to be used in SYN or SYN+ACK segment without negotiating with peer end system. In the example of Fig. 2, TCP options are sent with maximum segment size 
(MSS) option set 10 bytes, sack permitted option, timestamp option and window scale factor (WSF) option set 0 .

In the content part, the behaviors after establishing TCP connection are specified. In the each line of content part, the condition and the action are specified by being separated with semi-colon. In the condition, a trigger event and its parameter values are specified. There are three types of trigger events, recv, send and wait, which mean received packet, sending packet and timer expiration, as described above. In the case of recv and send events, several expressions such as "=", "<=", ">=", ">" and "<" can be used with a compared value. If any is specified as the value, the condition always becomes true as far as the parameter exists. At the end of the condition, varupd which means whether internal variables related to the received packet such as $r c v \_n x t$ are updated or not is also specified. If var-upd is OFF at recv event, it becomes the same situation that the received packet is lost at network. In the action, a packet to be sent with its parameter values at a true condition is specified. All of the parameter values in the TCP header except urgent pointer and checksum should be specified in the action. TCP options such as MSS and SACK are specified with parameter values if the options are included in the sent packet. At the end of the condition, var-upd which means whether internal variables related to the sent packet such as snd_nxt are updated or not is also specified. If no packet is desired to be sent, ignore is specified as action. If multiple packets are desired to be sent at a time, the second and later actions are specified without conditions.

Each line of content part in Figure 2 is interpreted as the following way:

line 1: If a packet with its sequence number (seq) equal to 1 is received, update the internal variables for the received packet, send a packet with its seq set 1, acknowledgment number (ack) set 11, flag bit (flag) set ack flag and window size (win) set 20 bytes and update the internal variables for the sent packet. Here, the value specified in seq or ack is relative one to the initial send sequence number or initial receive sequence number.

line 2: If a packet with seq equal to 11 is received, ignore the received packet without updating the internal variables.

line 3: If a packet with seq equal to 1 and ack equal to 11 is going to be sent, stop the packet being sent while updating the internal variables for the packet.

line 4: Wait one time slot (considered 500 millisecond) and send a packet with seq set 1, ack set 11, flag set ack flag and win set 30 bytes without updating internal variables for the packet.

line 5: If a packet with seq equal to 11 is received, ignore the received packet while updating the internal variables. 
line 6: If a packet with seq equal to 21 is received, ignore the received packet without updating the internal variables.

line 7-10: If a packet with $s e q$ equal to 31 is received, update the internal variables for the received packet, send 4 duplicate packets with its seq set 1 , ack set 11, the blocks for selective acknowledgment (sack) set 31-41, flag set ack flag and win set 30 bytes and update the internal variables for every sent packet.

line 11: If a packet with seq greater than 70 is received, update the internal variables for the received packet, send a packet with its seq set 1, flag set rst flag and win set 30 bytes and update the internal variables for the sent packet.

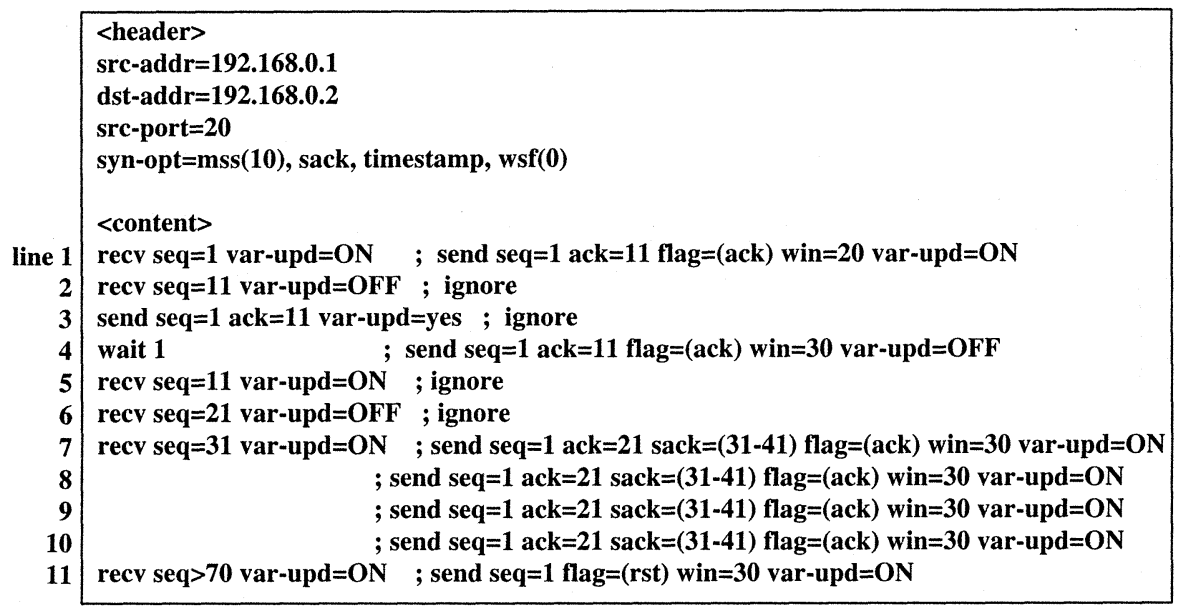

Figure 2. Example of Test Scenario.

\subsection{Scenario Interpreter}

The flow chart presenting how scenario interpreter works is illustrated in Fig. 3. Scenario interpreter maintains a test scenario loaded to the test system. It examines all packets which are received or going to be sent and checks whether the TCP connection of the packet is under test or not. If the connection is under test, the interpreter checks whether the TCP state is ESTABLISHED or not. If not, the packet is dealt as a normal TCP behavior. Only if syn-opt is specified in the header part of the test scenario and SYN or SYN+ACK segments is going to be sent, the TCP options for the sent packet are modified as what specified at syn-opt before sending it. If the state is ESTABLISHED, the interpreter maintains the scenario counter, which 
indicates the line executed currently. The counter starts at the first line of the content part when the TCP state enters ESTABLISHED and is incremented only when the condition at the counter is satisfied and the action is executed. If the counter reaches to the end, further events are dealt as normal TCP behavior. If the trigger event is wait, a timer is invoked. The expired time is set to the number of time slots specified in the condition. While the timer is working, the following sent or received events obey to normal TCP behavior. When the timer is expired, the action for the wait is executed and the scenario counter is incremented. Even if the counter reaches to the end, the communication under test does not finish at the time. It continues by normal TCP behavior.

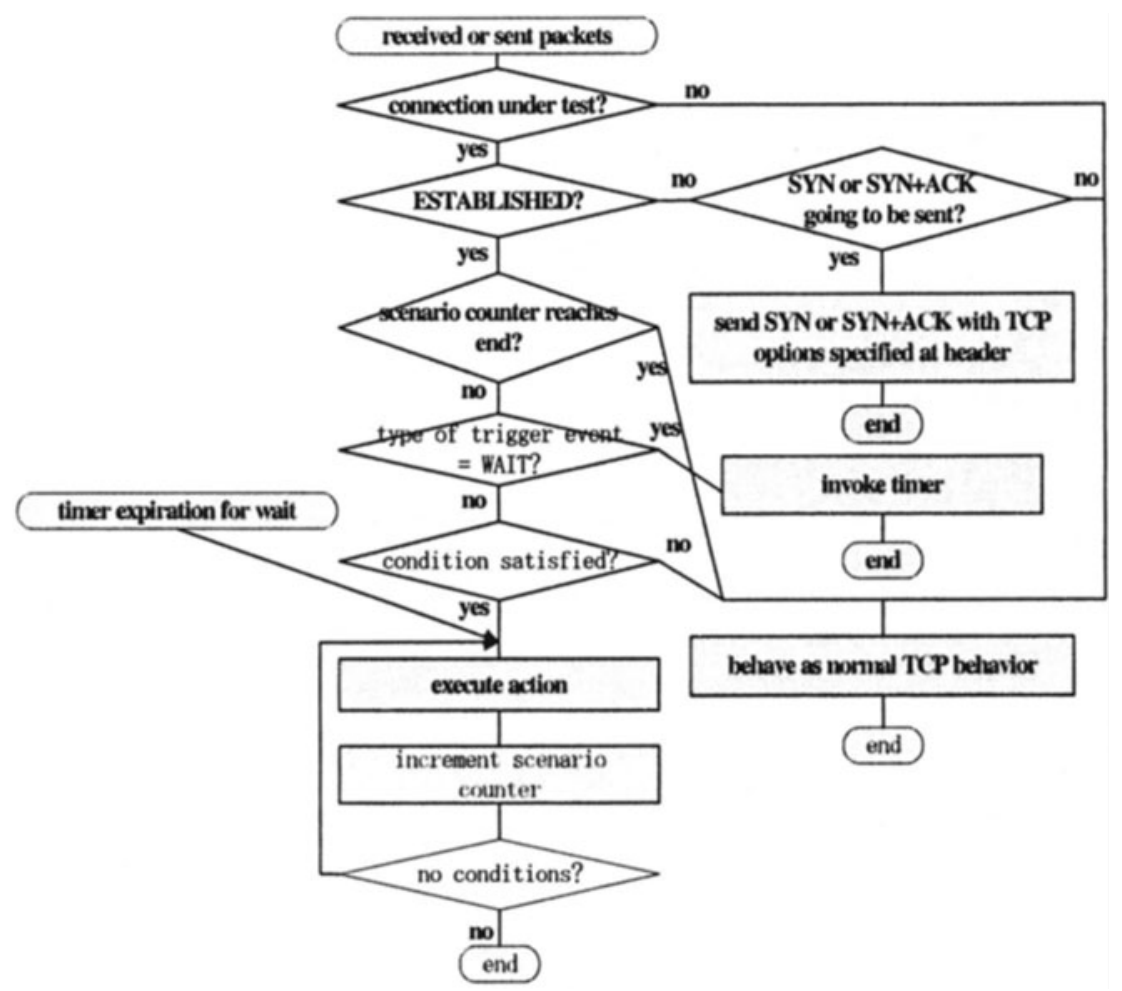

Figure 3. Flow Chart for Scenario Interpreter.

Figure 4 illustrates the sequence of communication when the test scenario in Fig. 2 is performed. The test causes the system under test (SUT) retransmit by timeout and fast retransmit. The scenario was written with a knowledge that SUT performs slow start with initial congestion window set 1. Therefore, SUT cannot send any packet after sending the first DATA 
segment of its seq 21, which causes retransmit by timeout. The test system sends 4 duplicate ACK segments illegally, which causes fast retransmit at SUT. The sequence includes a part behaving normally. In the part, DATA segments from 21 to 70 are sent and acknowledged. The test system does not need to specify this part in the test scenario.

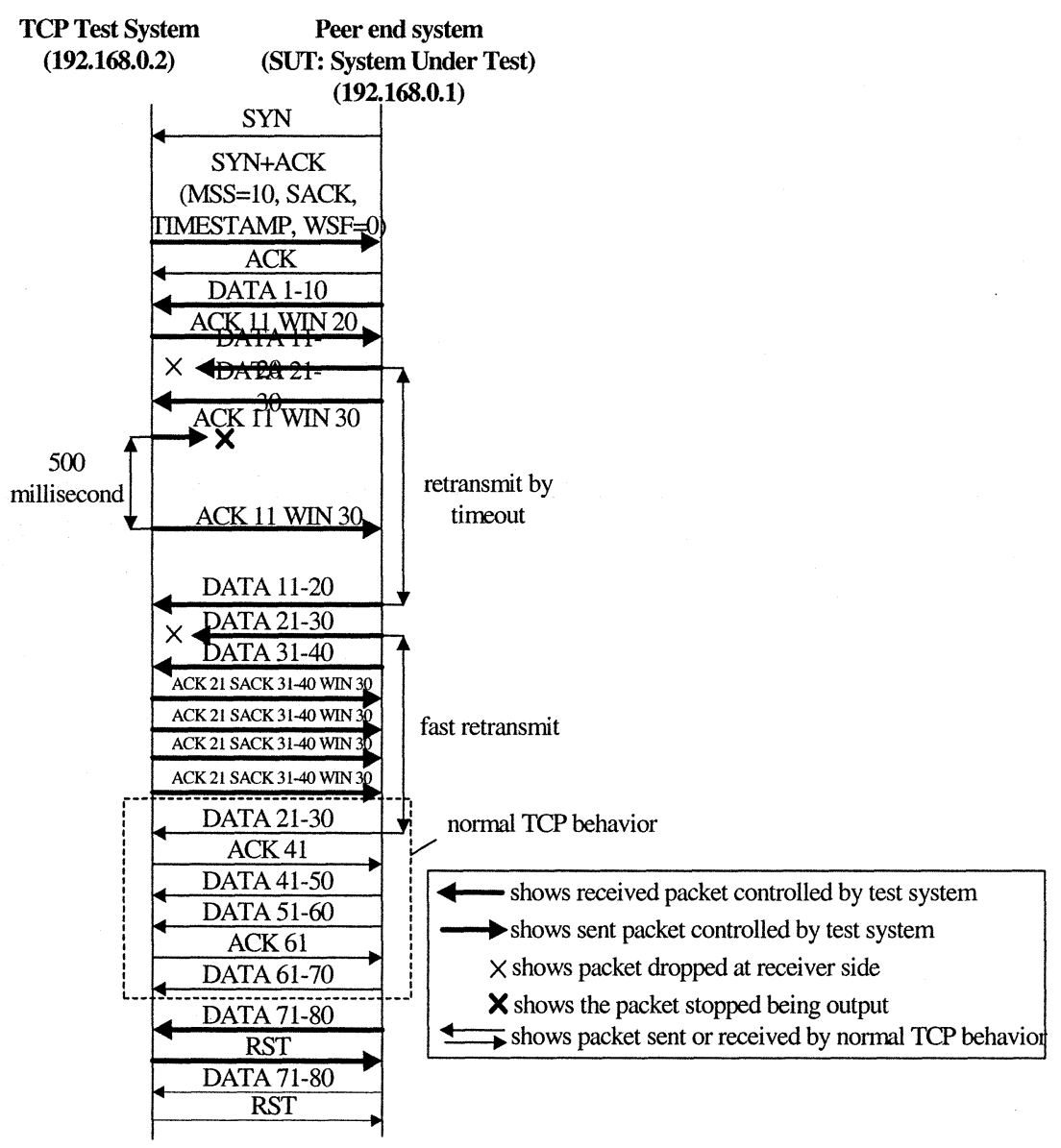

Figure 4. Example of Exceptional Packet Sequences.

\subsection{Log Collector}

Log collector saves a packet received or going to be sent to the communication log if the TCP connection of the packet is under test and the function is set on. Figure 5 shows a communication log collected at the test of Fig. 2. The output format is almost same as that of tcpdump [14]. The differences are as follows: 
- The communication log shows the scenario counter on the top of line for each event.

- The communication log can show the packets which are not actually sent or received at TCP of the test system using parenthesis such as line 2 and line 3.

- The communication log shows whether internal variables are updated or not. If updated, (var-upd) is added to the line.

\begin{tabular}{|c|c|}
\hline \\
\hline \multicolumn{2}{|c|}{$0: 0.000128$ SYN+ACK seq=1,783,630 ack $=1$ win $=32,768$ mss $=10$ sack-permitted timestatmp wsf $=0$} \\
\hline $0: 0.000233$ & ACK seq=1 ack=1 win=32,768 (var-upd) \\
\hline 1: 0.001733 & DATA seq=1 ack=1 win=32,768 len=10 (var-upd) \\
\hline 1: 0.001855 & ACK seq $=1$ ack $=11$ win $=20$ \\
\hline 2: 0.001934 & (DATA seq=11 ack=1 win=32,768 len=10) \\
\hline \multicolumn{2}{|c|}{ 3: $0.002018($ ACK seq $=1$ ack $=11$ win $=8,760)($ var-upd $)$} \\
\hline \multicolumn{2}{|c|}{ 4: 0.478445 ACK seq $=1$ ack $=11$ win $=30$} \\
\hline 5: 3.712842 & DATÁ seq=11 ack=1 win=32,768 len=10 (var-upd) \\
\hline 6: 3.712932 & $($ DATA seq $=21$ ack $=1$ win $=32,768$ len $=10)$ \\
\hline 7: 3.713015 & DATA seq $=31$ ack=1 win=32,768 len=10 (var-upd $)$ \\
\hline \multicolumn{2}{|c|}{ 7: 3.713099 ACK seq=1 ack=21 sack=(31-41) win=30 (var-upd) } \\
\hline \multicolumn{2}{|c|}{ 8: 3.713131 ACK seq $=1$ ack $=21$ sack $=(31-41)$ win $=30$ (var-upd) } \\
\hline \multicolumn{2}{|c|}{ 9: 3.713148 ACK seq=1 ack=21 sack=(31-41) win=30 (var-upd) } \\
\hline \multicolumn{2}{|c|}{ 10: 3.713166 ACK seq=1 ack=21 sack=(31-41) win=30 (var-upd) } \\
\hline 11: 3.713275 & DATA seq=21 ack=1 win=32,768 len=10 \\
\hline \multicolumn{2}{|c|}{ 11: 3.713383 ACK seq=1 ack=41 win=8,760 } \\
\hline 11: 3.713425 & DATA seq=41 ack=1 win=32,768 len=10 \\
\hline 11: 3.713499 & DATA seq $=51$ ack $=1$ win $=32,768$ len $=10$ \\
\hline \multicolumn{2}{|c|}{ 11: 3.713548 ACK seq=1 ack=61 win=8,760 } \\
\hline 11: 3.713552 & DATA seq=61 ack $=1$ win $=32,768$ len $=10$ \\
\hline 11: 3.713631 & DATA seq $=71$ ack $=1$ win $=32,768$ len $=10$ \\
\hline \multicolumn{2}{|c|}{ 11: 3.713693 RST seq=1 win $=30$ (var-upd $)$} \\
\hline end:3.713755 & DATA seq $=81$ ack $=1$ win $=32$, \\
\hline
\end{tabular}

Figure 5. Example of Communication Log.

\section{IMPLEMENTATION}

We implemented the TCP test system based on NetBSD 1.3.2 with SACK developed by Pittsburgh Supercomputing Center (PSC) [15] by modifying its source code. Scenario loader was implemented as a user program. Scenario interpreter and log collector were implemented in the kernel by modifying PSC's TCP module. For the interface for scenario loader to control the in-kernel module, we used setsockopt() function. The test scenario is loaded to TCP module through this function. The test scenario in the TCP module is deleted or referred to though the function. The test scenario is maintained as a global variable in the TCP module and 
copied to tcpcb structure [16] when connect() or accept() is invoked at a TCP connection under test. We modified the source codes related to TCP module in the following way in order to handle scenario interpretation and realize exceptional packet sequences:

- The source code for interpretation is newly added to the TCP module.

- In order to send exceptional packets specified in the test scenario, the packet in the action is constructed before tcp_output() is called.

- In order to interrupt the receiving and sending processes when the condition specified in the test scenario is satisfied, the codes for checking the condition is added to the top parts of tcp_input() and tcp_output().

- In order to avoid internal variables for updating when var-upd is OFF, tcp_input() and tcp_output() include the codes to skip the update.

- The wait function is realized in the same way as the system timer used for detecting retransmission timeout.

Through the implementation of the test system in the TCP module, it was difficult to stop all of disagreeable packets such as ACK for window update. The difficulty comes from the situation that the window update ACK is sent spontaneously. Actually, the window update ACK is sent when an application program reads received data. Therefore, the test system cannot avoid sending window update ACKs independently of test scenario. To cope with this situation, we provided following solutions:

- If we found a disagreeable window update ACK through the communication log after executing a test scenario, we added one line to the current test scenario which ignores the sent the ACK as described in Paragraph 3.2. Our test system is designed to facilitate such an incremental modification of test scenario because we need to specify only exceptional behavior.

- We provided another mode for the behavior of scenario interpreter, which does not output any packets as far as a packet is not described in the test scenario as an action. Therefore, this mode does not produce any window update ACK.

As a TCP application program, public tools such as ftp and ttcp can be used. Only the total size of data and the direction should be controlled by the test operator. As log analyzer, a simple text viewer is used. To realize the log collector, a system function syslog is used. The code to write the syslog is added to in-kernel TCP module. 


\section{1 evaluation of SACK implementation}

We evaluated SACK implementations in various operating systems using the implemented TCP test system. Figure 6 shows the network configuration of this test. We attached a network simulator between the test system and the system under test, and inserted one-second round trip delay in order to examine how the congestion control works. We used $f t p$ as application program and created a file of 16 Kilobytes at the system under test and sent the file to the test system. We tested the sender's behavior when ACK segments with SACK options (which we call SACK segments) are received. The operating systems we tested are SPARC Solaris 2.6 with SACK patch (which we call Solaris 2.6), Intel Solaris 8 (which we call Solaris 8), Linux kernel 2.4.2 (which we call Linux), NetBSD 1.3.2 with SACK code developed by PSC (which we call NetBSD), and Windows 98 Second Edition (which we call Windows 98). We performed three different tests described in the following subsections.

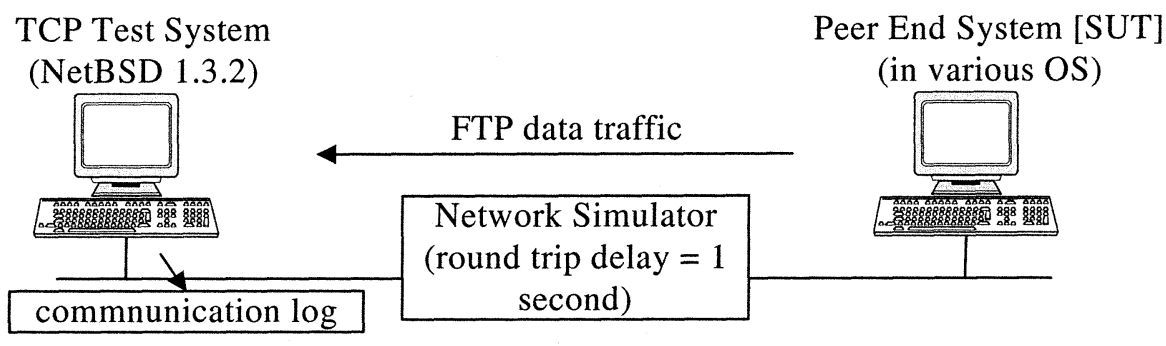

Figure 6. Network Configuration for SACK Testing.

\subsection{Multiple Drops in a Window}

SACK function notifies the sender with a range of dropped packets accurately, which is the same as that maintained by the receiver. If the sender implements the retransmission algorithm effectively, it can theoretically send all dropped packets in a window in one round trip time (RTT). Therefore, SACK function is effective between end systems over a link with long RTT such as satellite link. An approach to realize sender's behavior is proposed by [17] as pipe algorithm. We tested retransmission algorithm using the test scenario depicted in Fig. 7. This test scenario is very simple. It sets MSS as 100 bytes and emulates drops at network of every other packet after congestion window extends enough. Since the actions for 
received packets such as the packet with its seq 3501 , the test system sends SACK segments against each packet received in this RTT.

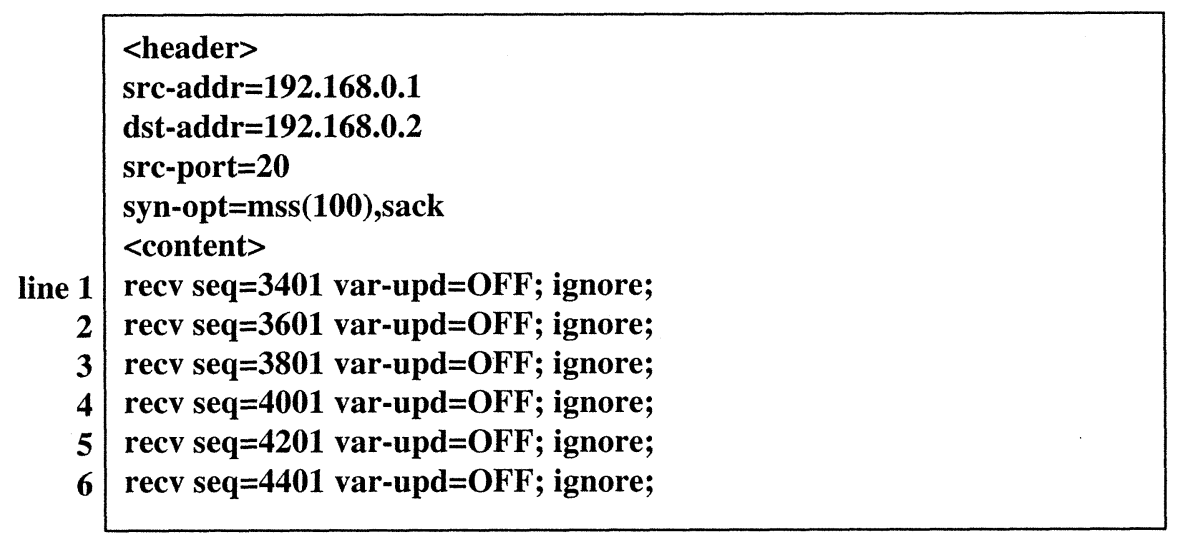

Figure 7. Test Scenario for Multiple Drops in a Window.

Table 1. Result for Multiple Drops in a Window

\begin{tabular}{|l|l|l|l|l|}
\hline OS & $\begin{array}{l}\text { Total Time } \\
\text { (second) }\end{array}$ & $\begin{array}{l}\text { Initial Congestion } \\
\text { Window }\end{array}$ & $\begin{array}{l}\text { number of } \\
\text { ACK }\end{array}$ & RTT \\
\hline Solaris 2.6 & 15.1 & 2 & 23 & 1 \\
\hline Solaris 8 & 14.1 & 4 & 23 & 2 \\
\hline Linux & 15.2 & 2 & 24 & 1 \\
\hline NetBSD & 16.1 & 2 & 23 & 1 \\
\hline Windows 98 & 17.3 & 2 & 24 & 3 \\
\hline
\end{tabular}

Table 1 shows the result of the test. Each column represents the operating system (OS), total elapsed time to transmit 16 Kilobytes, initial congestion window (ICW) used at slow start, number of ACK segments the SUT received before receiving duplicate ACK and RTT taken for retransmitting 6 dropped packets. Through this test, we found following facts and problems:

- All implementations deal with SACK options to retransmit dropped packets. If the retransmission algorithm for SACK were not implemented, it might take six RTTs to retransmit all of the dropped packets.

- Solaris 8 took two RTTs and Windows 98 took three RTTs to recover from dropped packets. However, we can not say the retransmission algorithms of these operating systems were worse than those of others. For example, when we set the ICW of Solaris 8 to 2, the RTT becomes one like others. From another test which drops 1501, 1701 and 1901, Linux took two RTTs while Solaris 2.6 and NetBSD took one. We think RTT taken to recover from multiple drops is highly related to ICW and 
number of ACK because these values decide the value of congestion window when the congestion occurs.

- We found typical implementations, which are inconsistent with [13], on Linux, Solaris 2.6 and Window 98. All of them seem to inflate window before receiving three duplicate ACK segments. In addition, Linux retransmits faster (by one duplicate $\mathrm{ACK}$ in this test) than other implementations when it receives SACK segments. Since Windows 98 sent only 96 bytes when it retransmitted the packet with its seq 4001, it took another one RTT to retransmit the rest of 4 bytes.

\subsection{Delay and Loss of SACK}

SACK segments may not be arrived in correct order to the sender. This situation is possible according to the network condition. Figure 8 shows the test for the sender's behavior when SACK segments are delayed and lost in network. In this figure, SACK segments for DATA with seq 3501 and 3901 are lost shown in line 2 and 6, and 3701 is delayed shown in line 4 and 9.

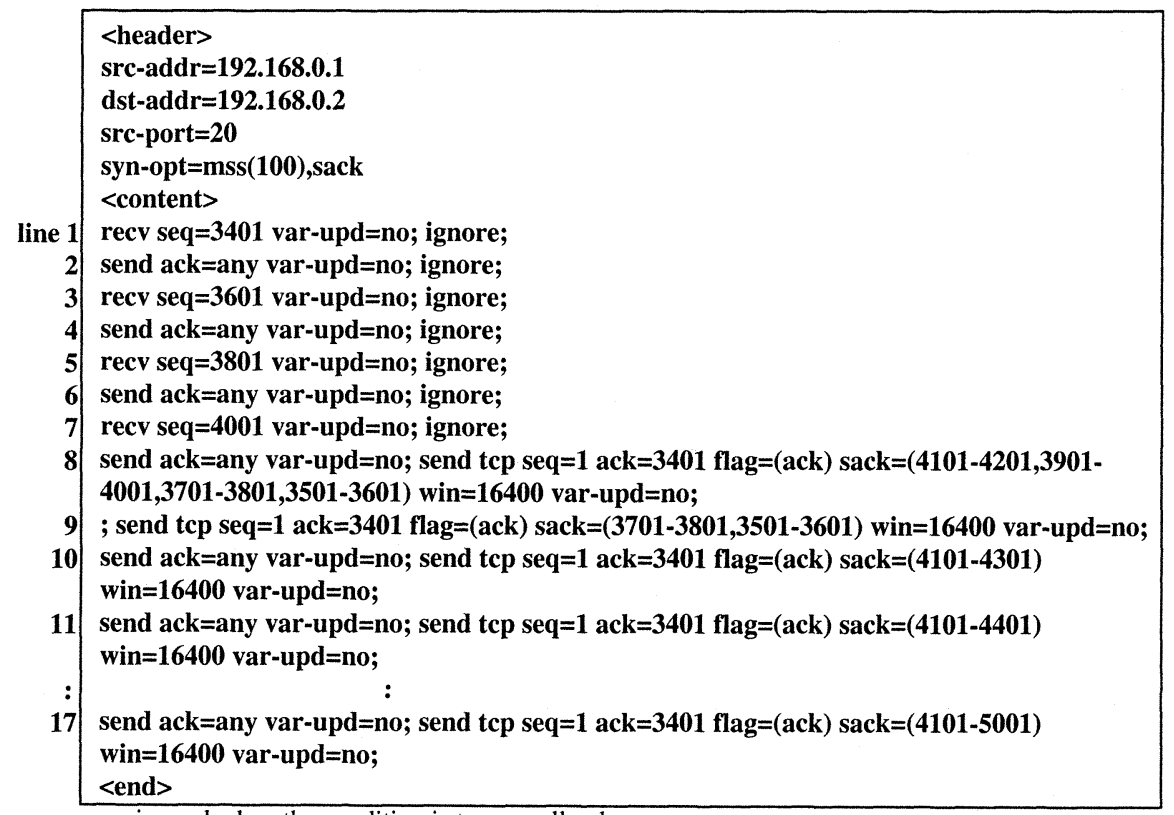

any is used when the condition is true on all value

Figure 8. Test Scenario for Delay and Loss of SACK Segments.

All implementations behaved well for these situations. The lost packets were retransmitted in one RTT in sequential order. We can estimate that 
current SACK implementations do not consider the order of arrival from this result.

\subsection{Duplicate and Identical SACK}

The third test we performed was sender's behavior to multiple duplicate ACK segments with duplicate and identical SACK. The literature [18] describes that a duplicate SACK segment is sent when the receiver receives a segment including the range of sequence number previously received. The format of duplicate SACK is the same as normal SACK specified in [4] except it uses older ranges of sequence number in SACK block relative to the acknowledgement number. Since the use of duplicate SACK was recently specified, it seems there were few implementations to support this. It is mentioned that Linux supports it. The duplicate ACK segment with the same SACK blocks, which we call identical SACK, should not be occurred as far as it is not duplicated in network in the current specifications. However, we think the sender should deal well the receipt of identical SACK. Figure 9 shows the test scenario for duplicate SACK.

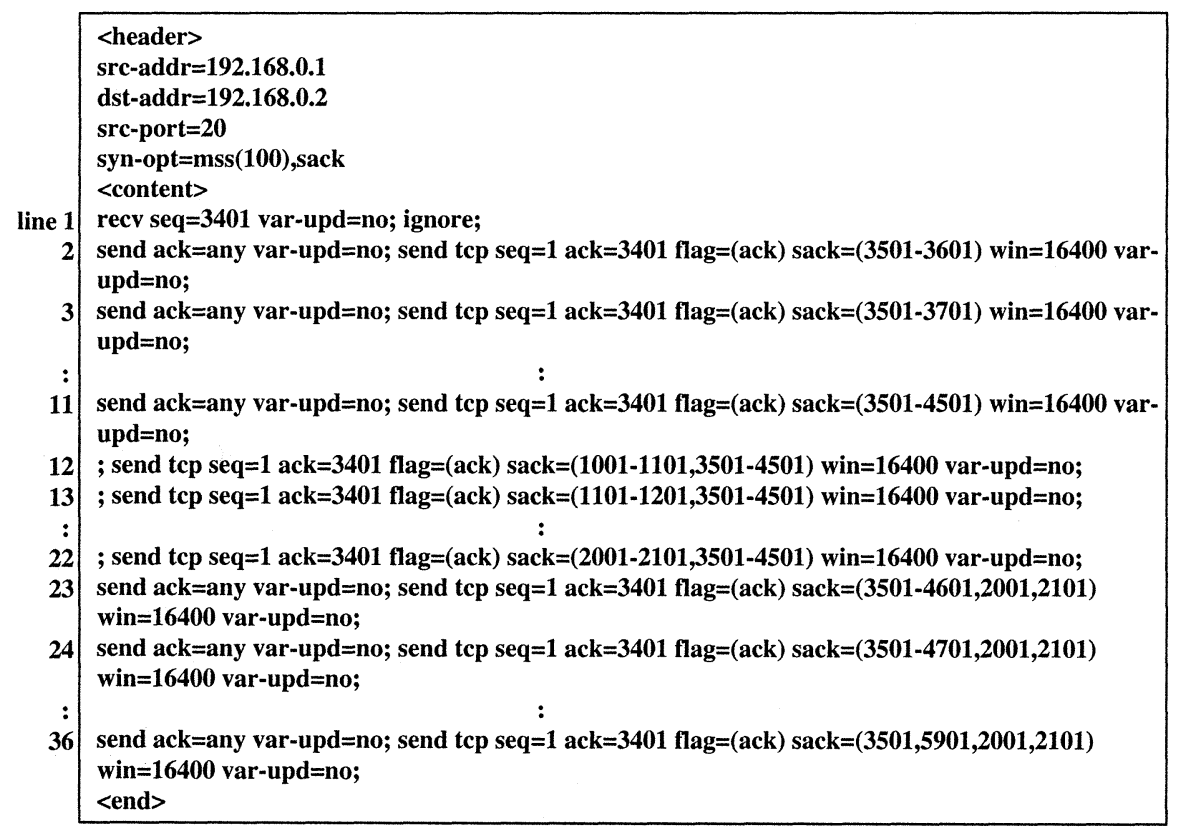

Figure 9. Test Scenario for Duplicate SACK.

The implementations other than Linux seem to inflate window when it receives duplicate or identical SACK. The behavior is not agreeable from the 
theory in the pipe algorithm. NetBSD decrements pipe value every time when it receives duplicate ACK without referring the values in SACK block by referring its source code. Solaris 2.6, Solaris 8 and Windows 98 seem to implement in the same way since the result in the communication log was the same. On the other hand, Linux did not inflate window to duplicate SACK. It maintains the bytes of unacknowledged segments instead of pipe value by referring the source code. The result for identical SACK was also the same as above.

\section{CONCLUSION}

In this paper, we described the design, implementation and experimental result of a TCP test system. The system is designed to reduce the burden of test operator by specifying only exceptional behaviors which the operator wishes to test in the test scenario among a complete communication. The system supports a variety of description of the test scenario. It provides three types of trigger events as the condition to cause the exceptional behavior, which are received packet, sending packet and timer expiration. The condition is described using parameters in the packet. As the action for an event, a packet with any parameter values can be produced. It is also possible not to send any packet for an action. The function above is useful for testing congestion control, which is one of the most important functions in TCP.

The scenario interpreter selects the action to each event by the condition described in the test scenario. If the condition is satisfied, the action described in the test scenario is executed. If not, the action is determined by the behavior of original TCP module.

The scenario interpreter and the log collector of the test system are implemented into the TCP module by modifying the module itself. It is implemented on NetBSD with SACK code developed by PSC. The test scenario is uploaded to the TCP module before it is executed. This implementation facilitates the combination of normal TCP behavior and scenario-oriented TCP behavior in a communication.

For the experimental usage of the system, we described the evaluation of SACK implementations on several operating systems. We selected test cases which can happen in actual environment. We found several facts and problems in SACK implementations in some operating systems. It is considered that the test system is effective on testing of TCP through this experiment.

This research is funded by TAO (Telecommunications Advancement Organization of Japan). 


\section{REFERENCES}

[1] W. Stevens, “TCP/IP Illustrated, Vol. 1: The Protocols," Addison Wesley, 1994.

[2] V. Paxson, M. Allman, S.Dawson, W.Fenner, J. Griner, I. Heavens, K. Lahey, J. Semke and B. Volz, "Known TCP Implementation Problems," RFC 2525, Mar. 1999.

[3] S. Floyd and T. Henderson, "The NewReno Modification to TCP's Fast Recovery Algorithm,” RFC2582, Apr. 1999.

[4] M. Mathis, J.Mahdavi, S.Floyd, and A. Romanow, "TCP Selective Acknowledgment Option," RFC 2018, Oct. 1996.

[5] S. Savage, N. Cardwell, D. Wetherall and T. Anderson, "TCP Congestion Control with a Misbehaving Receiver, " Proc. of SIGCOMM 1999, Aug. 1999.

[6] S. Parker and C. Schmechel, "Some Testing Tools for TCP Implementors," RFC 2398, Aug. 1998.

[7] V. Paxon, "Automated Packet Trace Analysis of TCP Implementations," in Proc. of SIGCOMM '97, Aug. 1997.

[8] T. Kato, T. Ogishi, A. Idoue and K. Suzuki, "Design of Protocol Monitor Emulating Behaviors of TCP/IP Protocols,” in Proc. of IWTCS '97, Sep. 1997.

[9] J. Padhye and S. Floyd, "On Inferring TCP Behavior," in Proc. of SIGCOMM '2001, Aug. 2001.

[10] Fyodor, "Remote OS Detection via TCP/IP Stack Fingerprinting," http://www.insecure.org/nmap/nmap-fingerprinting-article.html, Dec. 1998.

[11] R. Gecse and P. Krémer, "Automated Test of TCP Congestion Control Algorithm," in Proc. of IWTCS '99, Sep. 1999.

[12] "OSI - Open System Interconnection, Conformance testing methodology and framework," ISO/IEC 9646, 1997

[13] M. Allman, V. Paxson and W. Stevens, "TCP Congestion Control," RFC 2581, Apr. 1999.

[14] “tcpdump/libpcap Homepage," http://www.tcpdump.org.

[15] "Pittsburgh Supercomputing Center (PSC) Homepage," http://www.psc.edu/networking/tcp.html

[16] G. Wright and W. Stevens, “TCP/IP Illustrated, Vol. 2: The Implementation," Addison Wesley, Jan. 1995.

[17] K. Fall and S. Floyd, "Simulation-based Comparisons of Tahoe, Reno, and SACK TCP," SIGCOMM CCR, Jul. 1996.

[18] S. Floyd, J. Mahdavi, M. Mathis and M. Podolsky, "An Extension to the Selective Acknowledgement (SACK) Option for TCP,” RFC 2883, Jul. 2000. 